\title{
Correction to: Development of a tool for benchmarking of clinical pharmacy activities
}

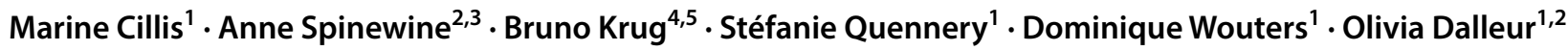

Published online: 29 November 2018

(c) Springer Nature Switzerland AG 2018

\section{Correction to: International Journal of Clinical Pharmacy https://doi.org/10.1007/s11096-018-0725-6}

In the original publication of an article, the corresponding author name has been swapped. Now the correct name has been published in this correction.

The original article has been corrected and can be found online at https://doi.org/10.1007/s11096-018-0725-6.

Marine Cillis

marine.cillis@uclouvain.be

1 Pharmacy Department, Université Catholique de Louvain, Cliniques Universitaires Saint-Luc, Avenue Hippocrate 10, Brussels, Belgium

2 Clinical Pharmacy Research Group, Université Catholique de Louvain, Louvain Drug Research Institute, Avenue E Mounier, 72 bte B1.72.02, Brussels, Belgium

3 Université Catholique de Louvain, CHU UCL Namur Site Mont-Godinne, Pharmacy, Rue Dr Gaston Therasse, Yvoir, Belgium

4 Université Catholique de Louvain, Institute of Health and Society, Clos Chapelle-aux-champs, 30 bte 30.15 , Brussels, Belgium

5 Université Catholique de Louvain, CHU UCL Namur Site Mont-Godinne, Quality and Safety Officer, Rue Dr Gaston Therasse, Yvoir, Belgium 